hep-th/9901012

\title{
Black Holes, Shock Waves, and Causality in the AdS/CFT Correspondence
}

\author{
Gary T. Horowitz 1 and N. Itzhaki 9 \\ Department of Physics \\ University of California, Santa Barbara, CA 93106
}

\begin{abstract}
We find the expectation value of the energy-momentum tensor in the CFT corresponding to a moving black hole in AdS. Boosting the black hole to the speed of light, keeping the total energy fixed, yields a gravitational shock wave in AdS. The analogous procedure on the field theory side leads to "light cone" states, i.e., states with energy-momentum tensor localized on the light cone. The correspondence between the gravitational shock wave and these light cone states provides a useful tool for testing causality. We show, in several examples, how the CFT reproduces the causal relations in AdS.
\end{abstract}

\footnotetext{
1 gary@cosmic.physics.ucsb.edu

2 sunny@solkar.physics.ucsb.edu
} 


\section{Introduction}

Most of the recent investigations of the correspondence between string theory in anti-de Sitter (AdS) space and conformal field theory (CFT) [1] have focused on the Euclidean regime. Lorentzian processes have just begun to be studied [2, 3]. We will be concerned with the question of how some basic causal relations in AdS are reproduced in the conformal field theory. In the process of answering this question, we are led to a new description of black holes in terms of the CFT, and an interesting connection between gravitational shock waves in AdS and novel states of the CFT which are localized on the light cone.

As an example of the type of situation we wish to analyze, consider two massless particles which come from infinity in AdS from the same direction, but at different times. It is clear that the later particle cannot influence the earlier one since it is entirely to its future. However, in the CFT, the "scale-radius duality" [4 suggests that a single massless particle that comes in from infinity should be described by a localized excitation that expands outward [3]. So two massless particles should be described by two excitations, but since the particles start at the same position at different times, the second excitation lies inside the light cone of the first. It is certainly not obvious why the presence of the second excitation cannot influence the further evolution of the first.

As a second example, suppose we consider two massless particles that come in at the same time, but from different directions. These particles should be described by two localized excitations in the CFT which start at different points in space at the same time. If the excitations are separated by a distance $c$, it is clear from the CFT that interactions between them cannot occur before a time $t=c / 2$, and are very likely thereafter. Is there an analogous statement on the AdS side? At first sight this seems unlikely since the two massless particles need not intersect, and even if they do, it is typically at a much later time.

We will resolve these puzzles below, and show that there is perfect agreement between the answers one obtains in the CFT and AdS. The first step is to realize that one must take into account the gravitational backreaction of the massless particles. For zero cosmological constant, the gravitational backreaction is given by the Aichelburg-Sexl metric [5] and describes a gravitational shock wave. This solution can be obtained by boosting the Schwarzschild metric with mass $M$ and taking the limit as $M \rightarrow 0, v \rightarrow 1$ keeping the total energy fixed. For negative cosmological constant, one can do exactly the same 
thing starting with the Schwarzschild anti-de Sitter metric. This was first done in four dimensions in [6] and is generalized to $d$ dimensions below (see also [7]). It is known that the Aichelburg-Sexl metric does not receive any $\alpha^{\prime}$ corrections [8, 9]. We will argue that this is also true for the gravitational shock wave in AdS.

To find the CFT dual of this gravitational shock wave, we start in section 2 by giving the field theory description of the Schwarzschild AdS black hole. Since only the AdS metric is excited on the supergravity side, the only nonzero expectation value in the CFT is the energy-momentum tensor. It turns out that the symmetries uniquely determine $<T_{\mu \nu}>$. This is straightforward when the CFT is defined on a sphere (cross time), but to describe the boosted black hole, it is more convenient to work on Minkowski space. So we find the form of $\left\langle T_{\mu \nu}>\right.$ in this case also.

In section 3, we perform the boost and construct the gravitational shock wave in AdS. Using the fact that on the CFT side the boosting corresponds to a Minkowski dilation, we find the expectation value of $T_{\mu \nu}$ in the CFT states which are associated with the gravitational shock wave. The result is an energy-momentum tensor which takes the form of null dust confined to the light cone. We will refer to these states as "light cone states".

In Section 4 we use the correspondence between the gravitational shock wave and these light cone states to show how the CFT reproduces some basic causal relations in AdS. In particular, we resolve the two puzzles mentioned above. The argument that the gravitational shock wave does not receive $\alpha^{\prime}$ corrections is given in section 5 . Section 6 contains a discussion of some extensions of our results, including the possibility of describing the formation of a black hole from the collision of two null particles in terms of the CFT.

The gravitational shock wave solutions in $A d S_{d}$ that we construct in section 3 might have other implications (which will not be explored here). For example, it may be used to calculate the amplitude for graviton exchange between two massless particles in AdS (in Minkowski space this was done in [10]) which is essential for the four point functions [11]. Also the shock wave solution in $A d S_{7}$ can be dimensionally reduced to construct the type IIA solution of D0-branes which are localized on D4-branes in the longitudinal and radial directions and are smeared along the angular directions. These solutions might be useful to study the $(0,2)$ theory living on M5-branes using the DLCQ approach [12, 13]. 


\section{CFT description of black holes}

Although most of our discussion applies equally well to AdS in various dimensions, to be specific, we will concentrate on the case of $A d S_{5} \times S^{5}$. String theory on this space is believed to be described by the $\mathcal{N}=4$ super Yang-Mills (SYM) theory [1]. Since the metric on $S^{5}$, and the self dual five form will remain unchanged, we will concentrate below on the metric on $A d S_{5}$.

We wish to find the field theory description of the Schwarzschild AdS black hole solution:

$$
d s^{2}=-\left(1+\frac{\tilde{r}^{2}}{R^{2}}-\frac{8 G M}{3 \pi \tilde{r}^{2}}\right) d \tilde{t}^{2}+\left(1+\frac{\tilde{r}^{2}}{R^{2}}-\frac{8 G M}{3 \pi \tilde{r}^{2}}\right)^{-1} d \tilde{r}^{2}+\tilde{r}^{2} d \Omega_{3}^{2}
$$

where $R=\left(4 \pi g_{s} N\right)^{1 / 4} l_{s}$ is the AdS radius and $G$ is the five dimensional Newton's constant. For finite $N$, the black hole will Hawking radiate and the exact description of the system is beyond our current abilities. In the large $N$ limit (for fixed $g_{s} N$ ), loop corrections are suppressed and the black hole becomes classical. Since $G \sim R^{3} / N^{2}$, to have a finite backreaction in the large $N$ limit, $M$ must be of the order $N^{2}$. Of course, we are used to classical black holes having finite mass. But this mass is in units where $G=1$. Since $G$ goes to zero in units of the AdS radius in the classical limit, we need $M$ to diverge. This implies that the corresponding field theory energies are of order $N^{2}$. Note that this is typical for near-extremal D-branes as well. That is, the energy above extremality of a near-extremal D-branes with finite horizon radius is proportional to $N^{2}$ [14, 15.

On the field theory side, we would like to characterize the states associated with classical black holes by the expectation value of certain operators. Since the solution (2.1) excites only the AdS metric, we conclude, using the arguments of [16, 17, 18], that the only non-vanishing expectation value is of the energy-momentum tensor. We claim that in this case, the symmetries determine completely the form of this expectation value. This is the field theory analog of the classical statement "black holes have no hair".

The AdS Schwarzschild solution (2.1) breaks the $S O(4,2)$ isometry of AdS to $S O(4) \times$ $S O(2)$. This can be seen explicitly by relating the above coordinates to the representation of AdS as the surface

$$
-T_{1}^{2}-T_{2}^{2}+X_{i}^{2}=-R^{2}, \quad i=1, \ldots, 4
$$


embedded in a six dimensional flat space with signature $(2,4)$. In this representation

$$
T_{1}=\left(R^{2}+\tilde{r}^{2}\right)^{1 / 2} \sin (\tilde{t} / R), \quad T_{2}=\left(R^{2}+\tilde{r}^{2}\right)^{1 / 2} \cos (\tilde{t} / R),
$$

and the $X_{i}$ are related to the spherical coordinates in the usual way. The $S O(2)$ rotation in the $T_{1}, T_{2}$ plane is simply translation in the $\tilde{t}$ direction. The $S O(4)$ isometries are rotations in the $X_{i}, X_{j}$ planes.

The SYM background associated with the black hole should have the same unbroken symmetries. In terms of the SYM theory on $S^{3} \times R$, this implies that the expectation value of $\tilde{T}_{\mu \nu}$ must be static and homogeneous.] Since $\tilde{T}_{\mu \nu}$ must also be tracefree, this uniquely fixes the energy-momentum tensor to be

$$
<\tilde{T}_{\mu \nu}>=\rho \hat{t}_{\mu} \hat{t}_{\nu}+\frac{1}{3} \rho h_{\mu \nu}
$$

where $\hat{t}_{\mu}$ is a unit vector in the time direction, $h_{\mu \nu}$ is the metric on $S^{3}$ with radius $R$, and $\rho=M / 2 \pi^{2} R^{3}$, is the mass density. This is not surprising for large $G M$, since large black holes are described by SYM states which are approximately thermal. However, the symmetries require that (2.4) is also valid for small $G M$. f

To describe boosted black holes, it is convenient to reexpress this energy-momentum tensor in terms of the theory on Minkowski space. The answer is not simply (2.4) with $h_{\mu \nu}$ denoting the flat metric on $R^{3}$. That would correspond to the near extremal three-brane geometry which has translational symmetry. We want to describe the static, spherically symmetric black hole in terms of the theory on $R^{3,1}$. There are two ways to do this. The first is to rewrite the unbroken $S O(2) \times S O(4)$ symmetries in terms of conformal symmetries of Minkowski space, and look for an energy-momentum tensor invariant under these symmetries. This can be done by writing AdS in the form

$$
d s^{2}=\frac{U^{2} \alpha^{\prime 2}}{R^{2}}\left(-d t^{2}+d x_{i}^{2}\right)+\frac{R^{2} d U^{2}}{U^{2}}
$$

where these coordinates are related to the embedding coordinates (2.2) via

$$
U=\frac{\left(X_{1}-T_{1}\right)}{\alpha^{\prime}}, \quad t=\frac{T_{2} R}{U \alpha^{\prime}}, \quad x_{i}=\frac{X_{i+1} R}{U \alpha^{\prime}}, \quad i=1,2,3 .
$$

\footnotetext{
${ }^{3}$ We use a tilde for the energy-momentum tensor on $S^{3} \times R$ to distinguish it from the one on Minkowski space below.

${ }^{4}$ Small black holes are known to be unstable to localizing on the $S^{5}$ [19], but if large $N$ SYM is really equivalent to supergravity, it should describe all solutions, even unstable ones. The shock wave we construct in the next section will be stable.
} 
The $\mathrm{SO}(3)$ subgroup of $\mathrm{SO}(4)$ implies that the SYM background is spherically symmetric. The remaining three generators of $S O(4)$ and the generator of $S O(2)$ are linear combinations of translations and special conformal transformations.

An easier way to proceed is to conformally map Minkowski space into $S^{3} \times R$ and use this to pull back the energy-momentum tensor (2.4). The required conformal factor can be derived by noticing that the metric on $S^{3} \times R$ is obtained by rescaling AdS in global coordinates by $R^{2} / \tilde{r}^{2}$ and taking the limit $\tilde{r} \rightarrow \infty$, while the metric on $R^{3,1}$ is obtained by rescaling AdS in coordinates (2.5) by $R^{2} / U^{2} \alpha^{\prime 2}$ and taking $U \rightarrow \infty$. So the two boundary metrics are related by the conformal factor:

$$
\omega^{2}=\lim _{U \rightarrow \infty} \frac{U^{2} \alpha^{\prime 2}}{\tilde{r}^{2}}=\frac{4 R^{4}}{\left(R^{2}+v^{2}\right)\left(R^{2}+u^{2}\right)},
$$

where we have written $\tilde{r}$ in terms of $\left(U, t, x^{i}\right)$ and set $u=t-r, v=t+r$, with $r^{2}=$ $x_{1}^{2}+x_{2}^{2}+x_{3}^{2}$. In other words, if we start with the Minkowski metric

$$
d s^{2}=-d u d v+\frac{(v-u)^{2}}{4} d \Omega_{2}^{2}
$$

and make the conformal transformation $g_{\mu \nu}=\omega^{2} \eta_{\mu \nu}$ (with $\omega$ given by (2.7)), we obtain

$$
d s^{2}=-4 R^{2} d \tilde{U} d \tilde{V}+R^{2} \sin ^{2}(\tilde{V}-\tilde{U}) d \Omega_{2}^{2}
$$

where

$$
u=R \tan \tilde{U}, \quad v=R \tan \tilde{V} .
$$

Defining $\tilde{V}=\frac{1}{2}\left(\frac{\tilde{t}}{R}+\theta\right)$ and $\tilde{U}=\frac{1}{2}\left(\frac{\tilde{t}}{R}-\theta\right)$ gives

$$
d s^{2}=-d \tilde{t}^{2}+R^{2}\left(d \theta^{2}+\sin ^{2} \theta d \Omega_{2}^{2}\right)
$$

which is just the metric on $S^{3} \times R$.

We now use the following fact (see, e.g., [20): If an energy-momentum tensor $T_{\mu \nu}$ is conserved and traceless with respect to a metric $g_{\mu \nu}$, then $\tilde{T}_{\mu \nu}$ is conserved and traceless with respect to $\tilde{g}_{\mu \nu}=\omega^{2} g_{\mu \nu}$ provided

$$
T_{\mu \nu}=\omega^{2} \tilde{T}_{\mu \nu}
$$

Using the conformal transformation (2.7), the coordinate change (2.10), and the known form of $<\tilde{T}_{\mu \nu}>$ (2.4), we obtain the following expectation value for the energy-momentum 
tensor on $R^{3,1}$

$$
\begin{aligned}
& <T_{u u}>=\frac{8 M}{3 \pi^{2}} \frac{R^{5}}{\left(R^{2}+u^{2}\right)^{3}\left(R^{2}+v^{2}\right)}, \\
& <T_{v v}>=\frac{8 M}{3 \pi^{2}} \frac{R^{5}}{\left(R^{2}+u^{2}\right)\left(R^{2}+v^{2}\right)^{3}}, \\
& <T_{u v}>=\frac{4 M}{3 \pi^{2}} \frac{R^{5}}{\left(R^{2}+u^{2}\right)^{2}\left(R^{2}+v^{2}\right)^{2}}, \\
& <T_{i j}>=\frac{8 M}{3 \pi^{2}} \frac{R^{5} \delta_{i j}}{\left(R^{2}+u^{2}\right)^{2}\left(R^{2}+v^{2}\right)^{2}},
\end{aligned}
$$

where $i$ and $j$ denote the orthonormal components on the two sphere.

Small black holes should approximately follow timelike geodesics. Consider the family of geodesics given (in the metric (2.5)) by

$$
\left(t^{2}+\Delta r^{2}\right) U^{2} \alpha^{\prime 2}=R^{4}
$$

The parameter $\Delta r$ labels different geodesics, and determines the maximum value of $U$ along the curve. The geodesic which stays at the origin $\tilde{r}=0$ in the global coordinates (2.3) corresponds to $\Delta r=R$. These geodesics are all related by a boost in the $T_{1}, X_{1}$ plane. It follows from (2.6) that such a boost corresponds to a dilation in Minkowski space. The boost which increases the maximum value of $U$ by $e^{\lambda}$, decreases distances in $R^{3,1}$ by $e^{-\lambda}$. The energy-momentum tensor of the boosted black hole can thus be obtained by simply rescaling the dimensionful constants $M$ and $R$. Setting $E=M e^{\lambda}$ and $\Delta r=R e^{-\lambda}$, we obtain

$$
\begin{aligned}
& <T_{u u}>=\frac{8 E}{3 \pi^{2}} \frac{\Delta r^{5}}{\left(\Delta r^{2}+u^{2}\right)^{3}\left(\Delta r^{2}+v^{2}\right)}, \\
& <T_{v v}>=\frac{8 E}{3 \pi^{2}} \frac{\Delta r^{5}}{\left(\Delta r^{2}+u^{2}\right)\left(\Delta r^{2}+v^{2}\right)^{3}}, \\
& <T_{u v}>=\frac{4 E}{3 \pi^{2}} \frac{\Delta r^{5}}{\left(\Delta r^{2}+u^{2}\right)^{2}\left(\Delta r^{2}+v^{2}\right)^{2}}, \\
& <T_{i j}>=\frac{8 E}{3 \pi^{2}} \frac{\Delta r^{5} \delta_{i j}}{\left(\Delta r^{2}+u^{2}\right)^{2}\left(\Delta r^{2}+v^{2}\right)^{2}} .
\end{aligned}
$$

One can verify that $E=\int d^{3} x<T_{00}>$, so it is indeed the field theory energy. It is related to the mass of the black hole by

$$
E=M e^{\lambda}=\frac{M R}{\Delta r}=\left.M \sqrt{g_{00}}\right|_{U_{\max }}
$$




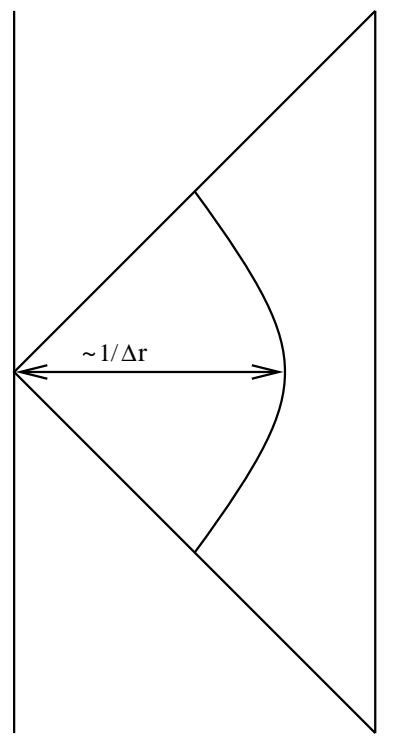

(a)

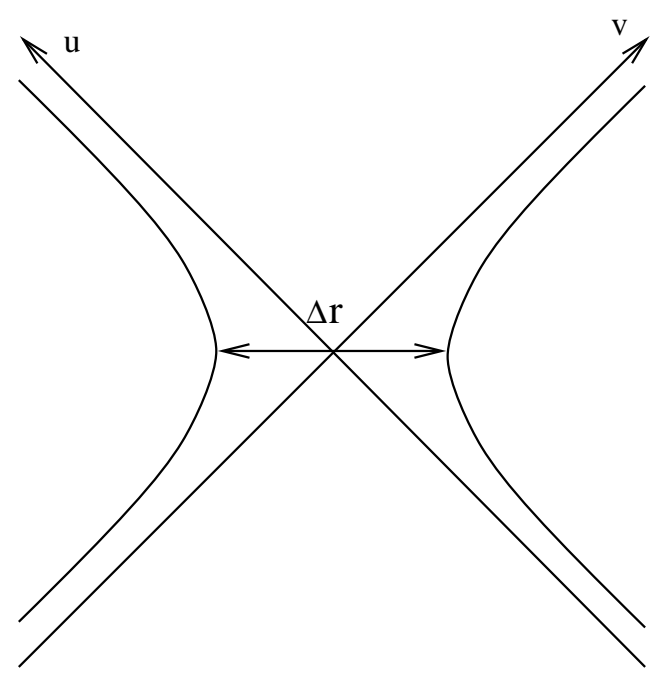

(b)

Figure 1: The UV/IR relation at work: (a) On the supergravity side a black hole is moving along a radial geodesic in AdS with maximal radial direction $\sim 1 / \Delta r$. (b) On the SYM side, the energy of the SYM state associated with the black hole is concentrated around the light cone with minimal size of $\Delta r$.

Let us describe the profile of the expectation values (2.15). The energy-momentum tensor is concentrated near the light cone $u=0$ or $v=0$ with a width of order $\Delta r$. So for $|t| \gg \Delta r$ the energy density extends over distances of the order of $t$ while for $|t| \ll \Delta r$ the energy density is spread over distances of the order $\Delta r$ (see fig. 1). This is in agreement with the UV/IR relation [4] since for $|t| \gg \Delta r$, eq. (2.14) implies that $U \sim R^{2} / t \alpha^{\prime}$ while for $|t| \ll \Delta r$ we get $U \sim R^{2} / \Delta r \alpha^{\prime}$ (see fig. 1 ).

\section{AdS shock wave solution and light cone states}

Having obtained the SYM description of the Schwarzschild AdS black hole, we now proceed to construct the analog of the Aichelburg-Sexl metric [5] describing a gravitational shock wave. On the supergravity side, this solution can be obtained in two ways. One approach is to boost the AdS black hole to the speed of light keeping the total energy fixed. Alternatively, one can obtain this solution using the method described in [21], which consists of appropriately gluing together two pieces of AdS spacetime along a null plane [7]. Using these approaches, the AdS shock wave metric was found in four dimensions in [6] and further studied in [22]. Below, we reexpress this solution in new coordinates which 
are more convenient for our purposes, and generalize it to higher dimensions. We then find the SYM description of this solution by applying the Minkowski dilation which is the analog of the AdS boost. To simplify the equations, in this section, we set $R^{2}=\alpha^{\prime}=1$.

To construct the gravitational shock wave we find it useful to work with the coordinate system

$$
y_{0}=\frac{T_{1}}{1+T_{2}}, \quad y_{i}=\frac{X_{i}}{1+T_{2}} .
$$

In these coordinates, the $A d S_{d}$ metric takes the form

$$
d s_{0}^{2}=\frac{4 \eta_{\mu \nu} d y^{\mu} d y^{\nu}}{\left(1-\eta_{\alpha \beta} y^{\alpha} y^{\beta}\right)^{2}}
$$

where $\eta_{\mu \nu}$ is the usual $d$ dimensional Minkowski metric. The physical spacetime corresponds to the region $\eta_{\mu \nu} y^{\mu} y^{\nu}<1$ and the boundary at infinity is $\eta_{\mu \nu} y^{\mu} y^{\nu}=1$. This clearly shows that $A d S_{d}$ is conformal to the region of $d$ dimensional Minkowski space lying inside a timelike hyperboloid. (This should not be confused with the $d-1$ dimensional Minkowski space of the field theory.) These coordinates do not cover the entire spacetime, but only the shaded region shown in Fig. 2a. The advantage of this coordinate system is that the metric is manifestly Lorentz invariant, though not static. This makes the form of the $A d S$ shock wave clearer knowing the shock wave solution in Minkowski space.

We would like to find the metric in the presence of a massless particle which moves along the null geodesic $y_{0}+y_{1}=0$. By analogy with the solution for zero cosmological constant, we try a metric of the form

$$
d s^{2}=d s_{0}^{2}+\frac{p \delta\left(y_{+}\right) f(\rho) d y_{+}^{2}}{\left(1+y_{+} y_{-}-\rho^{2}\right)}
$$

where

$$
y_{-}=y_{0}-y_{1}, \quad y_{+}=y_{0}+y_{1}, \quad \rho^{2}=\sum_{i=2}^{d-1} y_{i}^{2} .
$$

The reason for the factor $1+y_{+} y_{-}-\rho^{2}$ in the dominator is that it simplifies the equation for $f$. Substituting (3.3) into Einstein's equation $R_{\mu \nu}=-(d-1) g_{\mu \nu}$ yields the following linear equation for $f$ :

$$
D^{2} f-4(d-2) f=0
$$

where $D^{2}$ is the Laplacian on the transverse surface of constant $y_{-}$and $y_{+}=0$ which is just $d-2$ dimensional hyperbolic space with metric

$$
d s^{2}=\frac{d \rho^{2}+\rho^{2} d \Omega^{2}}{\left(1-\rho^{2}\right)^{2}} .
$$




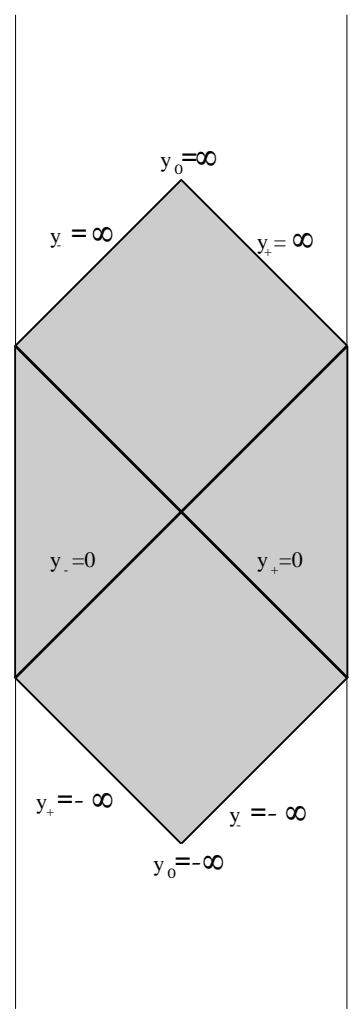

(a)

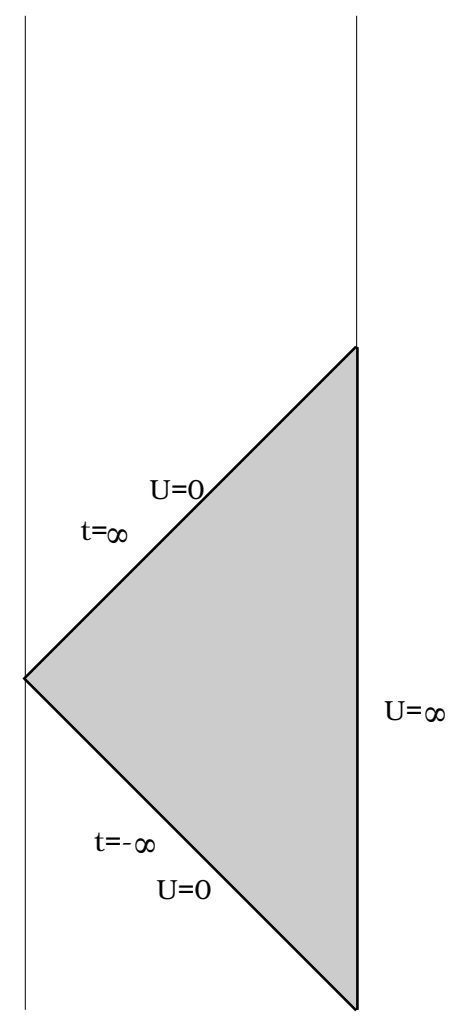

(b)

Figure 2: Regions of $A d S_{d}$ covered by different coordinates: The coordinates (3.1) cover the shaded region in (a). While the coordinates (2.6) cover the shaded region in (b). The matching between the coordinates is such that the line $U=0, t=\infty$ in (b) is the same as $y_{-}=0$ in (a).

To describe the field of a massless particle in AdS, one should add a delta function source to the right hand side of (3.5). Near $\rho=0$ the cosmological constant is negligible and the solution will resemble the Aichelburg-Sexl metric.

The fact that $f$ satisfies a linear equation implies that, like in Minkowski space, two parallel massless particles do not interact. That is, a massless particle moving along the geodesics $y_{+}=0, y_{i}=0$, for $i \neq 1$ will not interact with a massless particle moving along, say, $y_{+}=0, y_{2}=$ const., $y_{i}=0$ for $i \neq 1,2$.

When the cosmological constant is zero, the gravitational shock wave solution preserves half the supersymmetry [23]. We believe that the same is true for negative cosmological constant, although we have not yet checked this. The ability to superpose solutions is strong evidence for this.

We now turn to the SYM description of this gravitational shock wave. To relate string theory in $A d S$ to SYM on $R^{d-1}$ it is convenient to use the $\left(U, t, x^{i}\right)$ coordinates (2.6). The 
relation between these coordinates and $y^{\mu}(3.1)$ is

$$
U=-\frac{2 y_{-}}{1+y_{-} y_{+}-\rho^{2}}, \quad t=-\frac{1-y_{-} y_{+}+\rho^{2}}{2 y_{-}}, \quad x_{i}=-\frac{y_{i+1}}{y_{-}} .
$$

So the null geodesic $y_{+}=0, \rho=0$ is given by

$$
U=1 / t, \quad x_{i}=0, \quad \forall i
$$

We see that at $t=0$ the particle is located at the boundary and it falls towards $U=0$ at $t=\infty$.

In the previous section we found the SYM description of a particle (small black hole) which follows the geodesic (2.14). The geodesic (3.8) is obtained from (2.14) by taking the limit $\Delta r \rightarrow 0$. (Recall that we have set $R^{2}=\alpha^{\prime}$.) We want to take this limit keeping the energy fixed. Therefore, the field theory energy-momentum tensor associated with the null particle is obtained from (2.15) by taking the limit $\Delta r \rightarrow 0$ keeping $E$ fixed. It is easy to check that the total field theory energy, $E=\int d^{3} x<T_{00}>$, does not depend on $\Delta r$, so the limit is straightforward. It is clear that for $u, v \neq 0, \lim _{\Delta r \rightarrow 0}<T_{\mu \nu}>=0$. So the energy-momentum tensor is a delta-function supported the light cone, $u=0$ or $v=0$. Furthermore, on the future light cone, $u=0$, the only nonzero component is $\left\langle T_{u u}\right\rangle$. Similarly, on the past light cone, $v=0$, the only nonzero component is $\left\langle T_{v v}\right\rangle$. This implies that the energy-momentum tensor takes the form of null dust: $<T_{\mu \nu}>\propto l_{\mu} l_{\nu}$ where $l^{\mu}$ is a null vector tangent to the light cone. Since $\left\langle T_{\mu \nu}>\right.$ is nonzero only on the light cone, we will refer to these states as "light cone states".

We now review a field theory argument due to Coleman and Smarr [24 that supports our result that the energy-momentum tensor is localized on the light cone. Consider any classical field theory whose energy-momentum tensor satisfies

$$
\begin{aligned}
& T_{00} \geq 0, \\
& \partial_{\nu} T^{\mu \nu}=0, \\
& T_{\mu}^{\mu}=0 .
\end{aligned}
$$

From the moment of inertia $I(t)=\int d^{3} x r^{2} T_{00}$, one can define the average size of a state at a given time by

$$
\bar{r}(t)^{2}=I(t) / E, \quad \text { where } \quad E=\int d^{3} x T_{00} .
$$

Using eqs. (3.9, 3.10) one finds that $d^{2} \bar{r}^{2} / d t^{2}=2$ which implies that

$$
\bar{r}^{2}=t^{2}+\Delta r^{2}
$$


where $\Delta r$ is the minimum average size (which we assume occurs at $t=0$ ). This means that the initial configuration expands very rapidly, with its average size always larger than a sphere of light emitted at the origin at $t=0$. In the limit $\Delta r \rightarrow 0$, the initial configuration becomes localized at a point. Causality then requires that the fields vanish outside the light cone. Since $\bar{r}^{2}=t^{2}$, the energy density must be localized on the light cone.

The above argument was intended for a classical field theory. However the second two conditions of (3.9) are satisfied by the expectation value of the energy-momentum tensor in any conformal field theory. Furthermore, even though the first condition may be violated, as long as the total energy remains positive, we can still apply the argument. This is because a negative energy density will only decrease the average size $\bar{r}$, but the conclusion that $\bar{r} \geq t$ will still hold. Thus one can also apply this argument in the quantum theory. Even though the state is localized to a point at $t=0$, there is no contradiction with the uncertainty principle since it has essentially infinite energy: As discussed in the beginning of section 2, keeping a finite backreaction on the supergravity side in the classical limit $N \rightarrow \infty$ requires that $E \sim N^{2}$.

These light cone states are probably also supersymmetric. One usually thinks that supersymmetric states must be static, since the square of the supersymmetry generator is usually a time translation. The light cone states are clearly not static, but in a superconformal theory, one has the possibility that the supersymmetry generator will square to a special conformal transformation (see, e.g., [25]). One can verify that the light cone states are invariant under the special conformal transformations by translating the symmetries of the null geodesic (3.8) to the field theory.

We have been describing these states in terms of the field theory on Minkowski space. If one conformally rescales to $S^{3} \times R$, the light cone states start at a point, expand along the light cone to a maximal size at the equator, and then collapse back to a point at the other side of the sphere, where the particle again hits the boundary. Only the first half corresponds to the $R^{(3,1)}$ description. The second half can be described by a time reversed process on a second copy of the Minkowski space. 


\section{Tests of causality}

We now apply the correspondence between the gravitational shock waves and the light cone states to test whether SYM reproduces the causal relations in $A d S$. To begin, consider two massless particles in $A d S$ which come in from infinity from the same direction, but at different times. In other words, they follow the null geodesics $y_{+}=0, \rho=0$ and $y_{+}=a>0, \rho=0$. The first particle produces a gravitational field described by the shock wave metric (3.3). The second particle produces an analogous shock wave solution obtained from (3.3) by the coordinate change $y_{+} \rightarrow y_{+}-a$. Since the nontrivial curvature in the first shock wave is concentrated on the null plane $y_{+}=0$, and in the second is concentrated on $y_{+}=a$, we can clearly combine them into a single solution describing both particles. Since the shock waves do not intersect, there is no interaction between these particles. In the SYM theory, we have seen that the corresponding states are localized on the light cone, so once again, they do not interact (see fig. 3).

Note that the absence of interactions is a result of having massless particles. Suppose we consider, following [3], two strings which wind around the $x_{3}$ direction and come in from infinity from the same point (in the $x_{1}$ and $x_{2}$ directions) at different times. On the supergravity side, the backreaction of such strings is not confined to null surfaces and hence they will interact. On the field theory side, the wound strings are represented by flux tubes which expand in time. The expansion in this case is not confined to the light cone and hence the flux tubes will also interact.

Another case in which we know that there are no interactions on the supergravity side is of parallel massless particles. To be specific we consider two massless particles following the geodesics

$$
\begin{aligned}
& \text { I) } y_{+}=0, \quad y_{i}=0 \quad \forall i, \\
& \text { II) } \quad y_{+}=0, \quad y_{2}=\Lambda, \quad y_{i}=0 \quad \text { for } i \neq 2 .
\end{aligned}
$$

In the $\left(U, t, x^{i}\right)$ coordinates, these worldlines are described by

$$
\begin{aligned}
\text { I) } \quad t & =1 / U, \quad x_{i}=0, \\
\text { II) } \quad t & =A / U, \quad x_{1}=B / U, \quad x_{i}=0 \text { for } i>1,
\end{aligned}
$$

where $A=\frac{1+\Lambda^{2}}{1-\Lambda^{2}}$ and $B=\frac{2 \Lambda}{1-\Lambda^{2}}$. Notice that at the boundary $(U=\infty), t_{I}=0=t_{I I}$ and $x_{i I}=0=x_{i I I}$. In other words, even though the proper distance between the two particles 


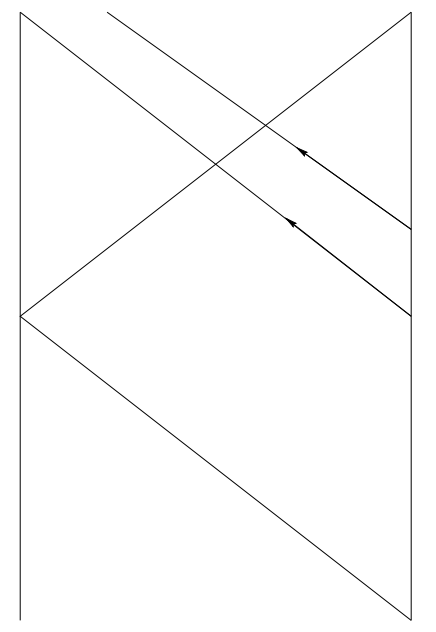

(a)

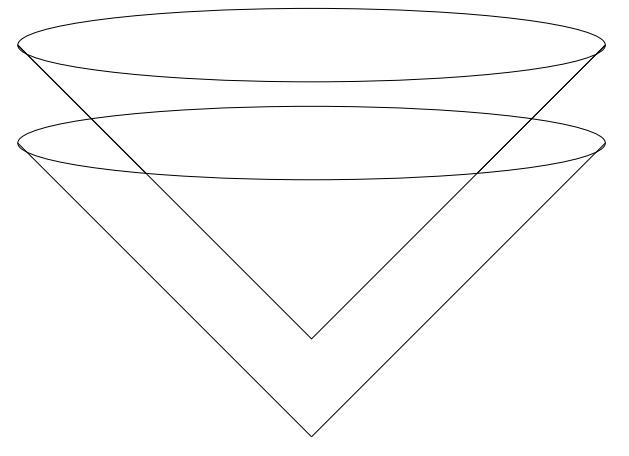

(b)

Figure 3: On the supergravity side (a) we have two shock waves which do not interact. On the SYM side (b) the states associated with these shock waves evolve on light cones which do not cross.

is constant in $A d S$ (as follows from (3.2)) their separation in $\left(t, x^{i}\right)$ goes to zero since $U^{2}$ blows up at the boundary. Thus from the SYM point of view, these two particles are described by states localized on the same light cone. It is certainly not obvious why they do not interact. Although we do not have a complete answer to this puzzle, this result can be made plausible as follows. Since $A^{2}-B^{2}=1$, the SYM states associated with the two particles are related to each other via a boost in the $\left(t, x_{1}\right)$ plane. (Note that the conclusion of the previous section that the states evolve on the light cone is invariant under the boost.) Classically, two massless particles starting from the same point but in different directions will not interact since they are not causally connected. The fact that the energy-momentum tensor of the light cone states takes the form of null dust strongly supports the idea that these states will not interact. If the light cone states are indeed supersymmetric and analogous to BPS states, this would provide another argument for the absence of interactions.

Next we consider a typical case in which there are interactions. We wish to compare the minimum time for interactions to occur in both the SYM and supergravity description. Consider two massless particles which come in from infinity at the same time, but from different directions in space. They are described by the null geodesics

$$
\text { I) } \quad U=1 / t, \quad x_{i}=0 \quad \forall i
$$




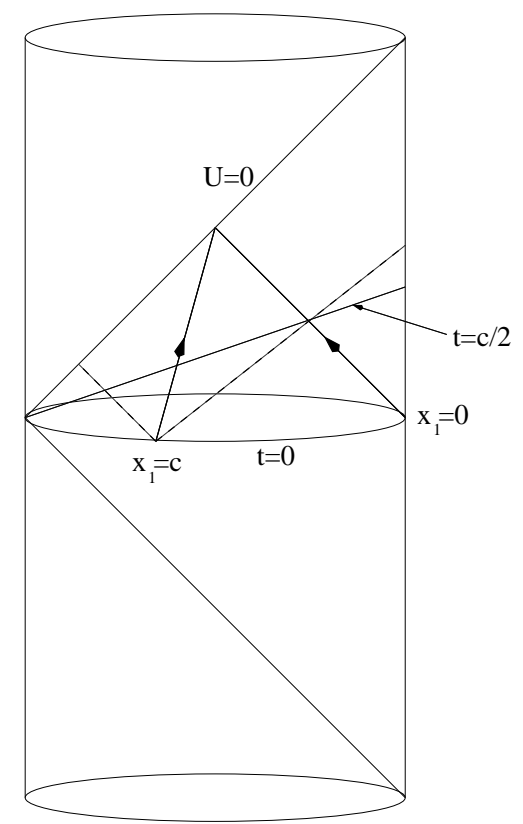

(a)

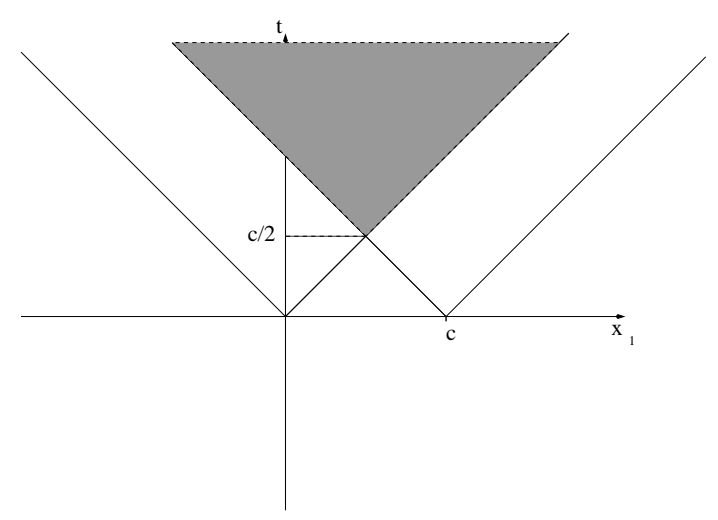

Figure 4: (a) The arrows indicate the trajectories of the massless particles. The dashed lines indicate the shock wave of the particle whose trajectory initiates at $x_{1}=c$. The shock wave of the other particle coincides (in the figure) with its trajectory. The shock waves cross each other at $t=c / 2$ which is in agreement with SYM (b).

$$
\text { II) } \quad U=1 / t, \quad x_{1}=c, \quad x_{i}=0 \text { for } i>1 \text {. }
$$

In terms of SYM we have two states which are evolving on the light cones

$$
\begin{aligned}
& \text { I) } \quad t^{2}-\sum_{i} x_{i}^{2}=0 \\
& \text { II) } \quad t^{2}-\left(x_{1}-c\right)^{2}-\sum_{i>1} x_{i}^{2}=0 .
\end{aligned}
$$

Clearly, these states will start to interact at a time $t=c / 2$ when the light cones intersect (see fig. 3b). This seems to contradict the fact that the particles in (4.3) do not intersect for any finite $t$. (The particles do intersect at $U=0$, but this corresponds to infinite $t$.) As we have discussed, the resolution is that the SYM state describes not only the motion of the particle but its gravitational back-reaction (that is, the shock wave) as well. We need to find, therefore, when the shock waves cross each other.

The exact solution for two intersecting shock waves is not known completely tunately, it is not needed to answer this question. The complicated part of the solution lies to the future of the intersection of the shock waves. Elsewhere, the solution is simply AdS with two approaching waves. We want to compute the time (in the $\left(U, t, x^{i}\right)$ coordinates

\footnotetext{
${ }^{5}$ For a discussion of the analogous solution with zero cosmological constant, see [26].
} 
associated with the Minkowski time in the field theory) for the shock waves to meet. However it is easier to describe the approaching shock waves in $y^{\mu}$ coordinates, since they lie along planes in these coordinates. So we will first use the $y^{\mu}$ coordinates, and then translate back using (3.7). We have seen that the solution for the gravitational field induced by particle (I) in (4.3) has a shock along the plane $y_{0}+y_{1}=0$. The region that overlaps the $\left(U, t, x^{i}\right)$ coordinates with $U>0$ has $-\infty<y_{0}<0$. Particle (II) follows the curve $y_{+}=c^{2} y_{-}, y_{2}=-c y_{-}, y_{i}=0$ for $i \neq 1,2$. This implies $y_{2}=2 c y_{1} /\left(1-c^{2}\right)$ so this worldline can be obtained from the original one (satisfying $y_{2}=0$ ) by a rotation in the $y_{1}, y_{2}$ plane. So the plane of its shock wave will be similarly rotated: $y_{0}+\cos \alpha y_{1}+\sin \alpha y_{2}=0$ where $\cos \alpha=\frac{1-c^{2}}{1+c^{2}}$. Therefore, the two shock waves will cross each-other and interact at

$$
-\infty \leq y_{0} \leq 0, \quad y_{1}=-y_{0}, \quad y_{2}=-c y_{0}
$$

and hence the field theory time of intersection is

$$
t \geq-\frac{1+y_{0}^{2} c^{2}}{4 y_{0}} \geq c / 2
$$

which is in a precise agreement with the SYM results.

This agreement actually reflects a deep connection between the causal structure of $A d S$ and its boundary. The conformally completed $A d S$ spacetime resembles a solid cylinder. But there is a crucial difference between the causal structure of this spacetime and a solid (timelike) cylinder in Minkowski spacetime. The intersection of a null plane in Minkowski spacetime and a timelike cylinder is a spacelike curve. Similarly, a null curve which stays on the boundary of the cylinder is not a null geodesic, and reaches the other side at a later time than a null geodesic which passes through the interior. In $A d S$ this is not the case. A null geodesic which starts on the boundary stays on the boundary. A null plane in the interior intersects the boundary at infinity in a null surface which is, in fact, the future light cone of a point $p$. In fact, the null plane is simply part of the future light cone of $p$ consisting of those null geodesics which enter the interior.

This explains the agreement we just found. The nontrivial curvature in the gravitational shock wave lies on a null plane which is part of the future light cone of a point on the boundary at infinity. So the intersection of two shock waves occurs first where their light cones intersect, which is on the boundary. Indeed, the time $t=c / 2$ corresponds to

\footnotetext{
${ }^{6}$ We could have said 'past light cone'. Since all null geodesics from a point $p$ on the boundary focus on the other side of the $S^{3}$ at the same point $q$, the future light cone of $p$ is the same as the past light cone of $q$.
} 
$y_{0}=-1 / c$ in (4.6) which implies $U=\infty$ using (4.5) and (3.7). Hence the time is the same as if one first restricts the light cones to the boundary and then asks when they intersect (which is just the field theory answer). Note that this argument is about the relation between the causal structure in the interior and on the boundary. The nontrivial curvature in the shock wave metric goes to zero for large distance from the source, so there is no 'extra curvature' on the boundary light cone. However, for any $t>c / 2$ the shock waves will intersect in the interior resulting in gravitational interactions.

\section{$5 \quad \alpha^{\prime}$ corrections}

In this section we show that there are no $\alpha^{\prime}$ corrections to the AdS shock wave solution. The fact that the solution probably breaks $1 / 2$ of the supersymmetries does not necessarily imply that there are no $\alpha^{\prime}$ corrections, since usually higher order corrections do modify BPS solutions (though they do not modify the relation between the charges and the energy). However, there are several examples when higher order terms do not modify the solution at all. Our argument for the AdS shock wave solution will be based on two such examples. The first is the $A d S_{5} \times S^{5}$ metric which has recently been shown to be an exact solution [27]. The second is the shock wave solution in Minkowski space.7 In [8] it was shown that this background is a solution of the $\sigma$-model equation of motion to all orders in perturbation theory. A geometrical derivation of this result, which rests on the fact that the curvature is null, was given in [9]. The result of [8] cannot be generalized to AdS since we do not know how to describe string theory with a RR-background by a $\sigma$-model. However, the geometrical approach of [9] can be generalized to AdS to show, together with the result of [27], that higher order (local) corrections will not modify the solution.

To see that there are no $\alpha^{\prime}$ corrections, let us write the AdS shock wave solution (3.3) in the form

$$
g_{\mu \nu}=g_{\mu \nu}^{0}+F l_{\mu} l_{\nu}
$$

where $g_{\mu \nu}^{0}$ is the AdS metric and $l_{\mu}=\partial_{\mu} y_{+}$. Then $l^{\mu} l_{\mu}=0$ and the Riemann curvature

\footnotetext{
${ }^{7}$ Another example, which is not related to our discussion, is of the SYM solution associated with of an electric charge, which is realized in string theory as a string attached to the D-brane. In [28] it was shown that the DBI corrections to SYM do not modify the solution.
} 
takes the form

$$
\begin{aligned}
& R_{\mu \nu \lambda \sigma}=R_{\mu \nu \lambda \sigma}^{0}+R_{\mu \nu \lambda \sigma}^{1} \\
& R_{\mu \nu \lambda \sigma}^{0}=-\left(g_{\mu \lambda} g_{\nu \sigma}-g_{\mu \sigma} g_{\lambda \nu}\right) \\
& R_{\mu \nu \lambda \sigma}^{1}=l_{[\mu} K_{\nu][\lambda} l_{\sigma]}
\end{aligned}
$$

$R_{\mu \nu \lambda \sigma}^{0}$ is the usual AdS curvature, and $K_{\mu \nu}$ is a symmetric tensor satisfying $K_{\mu \nu} l^{\nu}=0$. The $\alpha^{\prime}$ corrections are derived from terms in the action involving higher powers and derivatives of the curvature. Consider first a scalar constructed just from powers of the curvature. It is clear that $R_{\mu \nu \lambda \sigma}^{1}$ cannot contribute since the null vectors must contract either on the metric, on $K_{\mu \nu}$, or on themselves, and in all cases, the result is zero. Terms involving covariant derivatives of the curvature will also not contribute since the covariant derivative of $R_{\mu \nu \lambda \sigma}^{0}$ is zero and one can show that $\nabla_{\mu} l_{\nu}=V_{(\mu} l_{\nu)}$ for some vector $V_{\mu}$ which is orthogonal to $l^{\mu}$. Thus one cannot get rid of the factors of $l^{\mu}$ in $R_{\mu \nu \lambda \sigma}^{1}$ by taking its covariant derivative. Since covariant derivatives of $K_{\mu \nu}$ are also orthogonal to $l^{\mu}$, all contractions of $l^{\mu}$ will again vanish. Thus the only possible $\alpha^{\prime}$ correction terms come from $R_{\mu \nu \lambda \sigma}^{0}$. But these should be identical to the $\alpha^{\prime}$ corrections of AdS itself which have been shown to vanish [27. We conclude, therefore, that there are no $\alpha^{\prime}$ corrections to the AdS shock wave solution.

\section{Discussion}

We have found the description of a static spherically symmetric black hole in AdS in terms of the SYM theory on Minkowski space. By boosting the black hole to the speed of light keeping the total energy fixed, one obtains a gravitational shock wave in AdS. We have seen that the analogous procedure on the SYM side yields light cone states - states whose energy-momentum tensor is localized on the light cone. Using the duality between gravitational shock waves and light cone states, we have shown how the SYM reproduces some basic causal properties of AdS.

There are many open questions. In addition to the obvious one of testing other aspects of causality in AdS, one can ask if this analysis extends to the nonconformal dualities associated with other D-branes [29]. It should be possible to introduce gravitational shock waves in any supergravity background. Are there analogs of the light cone states in the corresponding field theories? 
One can imagine other applications of the duality between shock waves in AdS and light cone states. For example, the collision of two gravitational shock waves should produce a black hole. Since we know the SYM description of both the shock waves and the black hole, can one see this process in the SYM theory? In the example given in (4.3), the particles did not collide until an infinite field theory time, so one could never see the formation of a black hole in this case. However, one can consider other null geodesics which collide at finite time, such as

$$
\begin{aligned}
& \text { I) } t=\sqrt{1+\lambda^{2}} / U, \quad x_{1}=\lambda / U, \quad x_{i}=0 \text { for } i>1, \\
& \text { II) } t=\sqrt{1+\lambda^{2}} / U, \quad x_{1}=-\lambda / U+c, \quad x_{i}=0 \text { for } i>1,
\end{aligned}
$$

where $c>0, \lambda>0$. (The case $\lambda=0$ corresponds to (4.3).) These geodesics intersect in a time

$$
t=c \frac{\sqrt{1+\lambda^{2}}}{2 \lambda} .
$$

On the SYM side the two particles are described by two states evolving on the light cones (4.4). These light cones always intersect after a time $c / 2$ independent of $\lambda$. So the formation of a black hole is not directly related to this intersection. It seems to depend on concentrating the energy in the SYM. For $\lambda=0$ the energy density on each light cones is spherically symmetric before they intersect. The effect of $\lambda>0$ is to boost the light cone states toward each other, and hence concentrate the energy in the intersection region.

One might wonder how the energy-momentum tensor could ever evolve into the black hole form (2.15) which is nonzero everywhere, when causality requires that it be zero outside the future of the two light cones (4.4). The answer is simply that the collision of shock waves in AdS will take a long time to settle down to a static black hole. It is easy to see that this will not occur in a finite field theory time $t$. For large $\lambda$, one should be able to see the energy-momentum tensor start to approach the form (2.15) at late times.

It may be possible to make more progress on this question by considering the special case of $A d S_{3}$. One unusual feature of this case is that since the field equations require the solution to be locally $A d S_{3}$ away from the source, there is no gravitational shock wave solution. The solution describing a null particle in $A d S_{3}$ just has a conical singularity along a null geodesic [30]. It is not clear if this should be described by a $2 \mathrm{~d}$ analog of the light cone states, which would have support on two null curves. One advantage of the low dimensions is that it is possible to find an exact supergravity solution describing 
the formation of a black hole from the collision of two null particles [30. It would be interesting to find the CFT description of this process.

\section{Note added}

After this paper was written we learned of [31] which has some overlap with section 2.

\section{Acknowledgments}

We would like to thank A. Hashimoto, V. Hubeny, J. Polchinski and E. Witten for discussions. This work was supported in part by NSF grants PHY95-07065 and PHY9722022 .

\section{References}

[1] J.M. Maldacena, The large $N$ limit of superconformal field theories and supergravity, hep-th/9711200, Adv. Theor. Math. Phys. 2 (1998) 231.

[2] V. Balasubramanian, P. Kraus, and A. Lawrence, Bulk vs. Boundary Dynamics in Anti-de Sitter Spacetime, hep-th/9805171; V. Balasubramanian, P. Kraus, A. Lawrence and Sandip P. Trivedi, Holographic probes of anti-de Sitter space-times, hep-th/9808017; E. Keski-Vakkuri, Bulk and boundary dynamics in BTZ black holes, hep-th/9808037.

[3] T. Banks, M. R. Douglas, G. T. Horowitz and E. Martinec, ADS dynamics from conformal field theory, hep-th/9808016.

[4] L. Susskind and E. Witten, The holographic bound in anti-de sitter space, hepth/9805114.

[5] P.C. Aichelburg and R.U. Sexl, On the gravitational field of a massless particle, Gen. Rel. Grav. 2 (1971) 303.

[6] M. Hotta and M. Tanaka, Shock-wave geometry with non-vanishing cosmological constant, Class. Quantum Grav. 10 (1993) 307. 
[7] K. Sfetsos, On gravitational shock waves in curved space-times, hep-th/9408169, Nucl. Phys. B436 (1995) 721.

[8] D. Amati and C. Klimcik, Nonperturbative computation of the Weyl anomaly for a class of nontrivial backgrounds, Phys. Lett. B219 (1989) 443.

[9] G.T. Horowitz and A.R. Steif, Space-time singularities in string theory, Phys. Rev. Lett. 64 (1990) 260.

[10] G. 't Hooft, Graviton dominance in ultrahigh-energy scattering, Phys. Lett. B198 (1987) 61.

[11] D.Z. Freedman, S.D. Mathur, A. Matusis and L. Rastelli, Comments on 4 point functions in the CFT/AdS correspondence, hep-th/9808006.

[12] O. Aharony, M. Berkooz and N. Seiberg, Light cone description of (2,0) superconformal theories in six-dimensions, Adv. Theor. Math. Phys. 2 (1998) 119, hepth/9712117.

[13] H. Awata and S. Hirano, $A D S_{7} / C F T_{6}$ correspondence and the matrix models of M5branes, hep-th/9812218.

[14] I.R. Klebanov and A.A. Tseytlin, Entropy of near-extremal black p-branes, Nucl. Phys. B475 (1996) 164.

[15] I.R. Klebanov and L. Susskind, Schwarzschild black holes in various dimensions from matrix theory, Phys. Lett. B416 (1998) 62, hep-th/9709108.

[16] S.S. Gubser, I.R. Klebanov, and A.M. Polyakov, Gauge theory correlators from noncritical String Theory, hep-th/9802109, Phys. Lett. B428 (1998) 105.

[17] E. Witten, Anti-de Sitter space and holography, hep-th/9802150, Adv. Theor. Math. Phys. 2 (1998) 253.

[18] S. Ferrara, C. Fronsdal and A. Zaffaroni, On $N=8$ supergravity in $A d S_{5}$ and $N=4$ superconformal Yang-Mills theory, Nucl. Phys. B532 (1998) 153, hep-th/9802203.

[19] R. Gregory and R. Laflamme, Instability of charged black strings and P-branes, Nucl. Phys. B428 (1994) 399. 
[20] R.M. Wald, General relativity, The University of Chicago press (1984) p. 448.

[21] T. Dray and G. 't Hooft, The gravitational shock wave of a massless particle, Nucl. Phys. B253 (1985) 173.

[22] J. Podolsky and J. Griffiths, Impulsive waves in de Sitter and anti-de Sitter spacetimes generated by null particles with an arbitrary multiple structure, Class. Quant. Grav. 15 (1998) 453.

[23] K.P. Tod, All metrics admitting supercovariantly constant spinors, Phys. Lett. B121 (1983) 241.

[24] S. Coleman and L. Smarr, Are there geon analogues in sourceless gauge-field theories?, Commun. Math. Phys. 56 (1977) 1.

[25] S. Minwalla, Restrictions imposed by superconformal invariance on quantum field theories, hep-th/9712074.

[26] P. D'Eath and P. Payne, Gravitational radiation in black-hole collisions at the speed of light, Phys. Rev. D46 (1992) 658.

[27] R. Kallosh and A. Rajaraman, Vacua of $M$ theory and string theory, hep-th/9805041.

[28] C.G. Callan and J.M. Maldacena, Brane death and dynamics from the Born-Infeld action, Nucl. Phys. B513 (1998) 198, hep-th/9708147.

[29] N. Itzhaki, J. M. Maldacena, J. Sonnenschein and S. Yankielowicz, Supergravity and The large $N$ limit of theories with sixteen supercharges, Phys. Rev. D58 (1998) 046004, hep-th/9802042.

[30] H. J. Matschull, Black hole creation in 2+1 dimensions, gr-qc/9809087.

[31] U. H. Danielsson, E. Keski-Vakkuri and M. Kruczenski, Vacua, propagators, and holographic probes in AdS/CFT, hep-th/9812007. 